\title{
Impact of Nesting Methods on Model Performance
}

\author{
U. Bungert (1), K. Heinke Schlünzen (2)
}

Meteorological Institute, ZMAW, University of Hamburg, Bundesstr. 55, 20146 Hamburg, Germany

1) phone: +49-40-42838 5762, fax: +49-40-42838-5452

ursula.bungert@zmaw.de

2) phone: +49-40-42838 5082, fax: +49-40-42838-5452

heinke.schluenzen@zmaw.de

\section{Introduction}

The air quality directive of the European commission demands maps on concentrations and exceedances in different detail. For this purpose numerical models can be used. Some model systems are already adjusted to deliver the corresponding maps. For instance, the model system M-SYS consists of three mesoscale and one microscale model areas and applies one-way-nesting for meteorology and chemistry (Trukenmüller et al., 2004).

To calculate concentration data, reliable meteorological fields are needed, which should be calculated with the same resolution as the concentration maps. Lenz et al. (2000) have shown that the concentration fields are more sensitive to the description of the meteorological fields at the lateral boundaries than to the concentration fluxes over these boundaries. Nesting the meteorological fields, corresponding to the use of meteorology model results received on a coarser grid as time-dependent boundary values, needs to prescribe the boundary values as realistic as possible. In this work, we consider only the nesting of the meteorological fields and the influence of different update intervals of the forcing data on the model performance of nested simulations.

\section{Method}

The multiscale meteorology and chemistry model system M-SYS consists of the mesoscale models METRAS (MEsoscale TRAnsport and Stream model; Schlünzen, 1990; Schlünzen and Katzfey, 2003) and MECTM (Mesoscale Chemistry Transport Model; Müller et al., 2000; Schlünzen and Meyer, 2007), which are used in different resolutions, and the obstacle-resolving microscale models MITRAS and MICTM (Schlünzen et al., 2003; Lopez et al., 2006). Meteorology and chemistry transport are coupled off-line. Because the same grid, the same model physics and parameterizations are used, no interfaces are needed for the meteorology-chemistry coupling. Model results are, beside others, maps of concentration fields on different scales with different resolutions.

Although, the nesting is applied in M-SYS every time step, the forcing fields are only available at time intervals that are much longer than the time step used in the model. During these intervals, the forcing fields are linearly interpolated. To investigate, if the linear interpolation represents the development of the atmospheric fields in a realistic 
way, the mesoscale atmospheric model METRAS is used in different resolutions: The results of coarser grid simulations are used as forcing fields for the nested simulations in higher resolution. The time interval for writing the coarser model results determines the interval in which the forcing fields in the higher resolution simulations can be updated. By using the same model for calculating forcing data and performing the nested simulations on a higher resolving grid it is possible to perform well-controlled sensitivity studies on the effect of update interval on high resolution model results.

For nesting of meteorology models and for coupling of meteorology and chemistry transport it is important to know, how often the atmospheric forcing fields should be updated to sufficiently represent the non-linear processes triggered from the boundaries. If the update interval for the forcing fields is very small, the non-linear processes in the atmosphere should be well represented during these short intervals where a linear interpolation is applied. By continuous updating we eventually receive a model with multiple grids, which is a valid (but expensive) approach. However, despite costs this approach is not always possible, since limited area models need at some point lateral boundary values. At the outermost domain the forcing data are only available at specific time intervals. It needs to be known in which frequency these forcing data should be available. In addition, reading of forcing data needs additional time on the computer and thus, should be reduced to the necessary amount. Data update should be performed as much as necessary and as little as possible.

Changes in the atmospheric fields happen on different time scales and with different speed. Therefore, we adapt the time intervals for writing the model results (that are the forcing data for nested simulations) to the time scales, in which the atmospheric fields change, instead of using short but constant time intervals. If significant changes happen on short time scales, the results should be written more often than for more or less steady conditions.

The horizontal wind components, potential temperature and specific humidity are used in METRAS as forcing fields. Changes in the scalar quantities temperature and humidity are mainly induced by advection and diffusion, i.e. processes that depend on the wind. Therefore, we define the conditions for writing the model results only in dependence of changes in the horizontal wind components. The model results were alternatively written in regular intervals (3 hours, 6 hours), if the acceleration (changes in velocity) at $80 \%$ of the grid points is less than $5^{*} 10^{-5} \mathrm{~m} \mathrm{~s}^{-2}$, or if the acceleration (changes in velocity) at $20 \%$ of the grid points is less than $5 * 10^{-6} \mathrm{~m} \mathrm{~s}^{-2}$.

\section{Simulation set-up}

The outlined method of a situation-controlled writing of the model results is applied to a period in August 2003. During the simulated period (29-31 August 2003), a trough was situated from Spain to the Arctic northeast of Finland, lying between a high-pressure system over the Atlantic Ocean and another high-pressure system over South Eastern, later Eastern Europe. In between this trough, a small scale low has developed, that was 
lying over Belgium at the $28^{\text {th }}$, and then passed in West-East-direction over Northern Germany. Caused by this low, significant precipitation occurred in Northern Germany.

The model areas used for simulating this case are shown in Figure 1. The large area (Figure 1a) covering significant parts of Europe has a horizontal resolution of $18 \mathrm{~km}$, while the grid size in the small area (Figure 1b) is $6 \mathrm{~km}$. Model simulations are started for 20 CET, 28.08.2003. Comparisons are performed starting 1 hour after simulation begin (from 21 CET, 28.08.2003).

a)

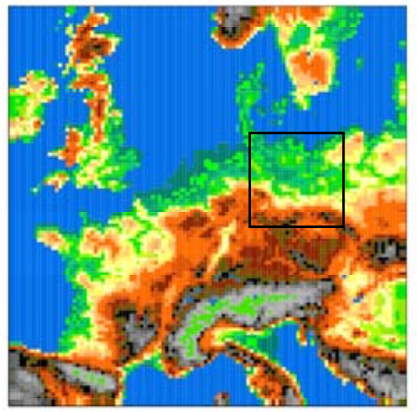

b)

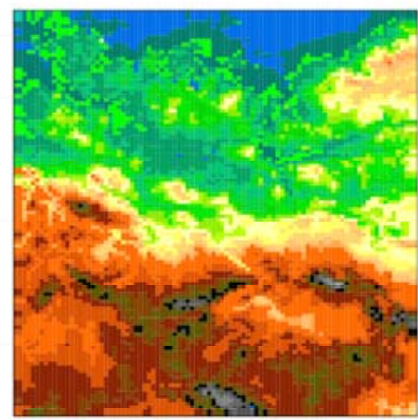

Figure 1: The model areas used for the coarse grid run yielding the forcing data (a) and for the nested simulations for the case "low pressure system over Europe“. The positioning of the nested area is shown by the frame in (a).

Several simulations on the $18 \mathrm{~km}$ grid that use different conditions for writing the model results produce the forcing data for the nested simulations that use a $6 \mathrm{~km}$ resolution. The $6 \mathrm{~km}$ resolution simulations are summarized in Table 1.

Table 1: Simulations performed with the high-resolution model area shown in Figure $1 b$.

\begin{tabular}{|c|c|c|}
\hline simulation name & forcing data from & Update interval \\
\hline eu_6km_3h & coarse grid METRAS run & 3 hours \\
\hline eu_6km_6h & coarse grid METRAS run & 6 hours \\
\hline eu_6km_lcout80 & coarse grid METRAS run & depending on results (80\%) \\
\hline eu_6km_lcout20 & coarse grid METRAS run & depending on results (20\%) \\
\hline eu_6km_ana & analysis & 12 hours \\
\hline eu_6km_nonesting & ---- & ---- \\
\hline
\end{tabular}

As can be seen from Table 1, besides the four simulations that are nested in METRAS $18 \mathrm{~km}$ results, two additional simulations were performed. In the simulation eu_6km_ana the forcing fields are derived from analyses that are available every 12 hours; thus a very large update interval is used. This update interval cannot be changed. Simulation eu_6km_nonesting uses no nesting and no heterogeneous initialisation, but only integrates the initial profile forward in time. This is a sensitivity study to allow evaluating, if the large-scale situation has any impact on the high-resolution results.

In the following we compare results of the all simulations given in Table 1 to each other and to a reference case. This reference case is a simulation with high resolution in the whole domain (Figure 1a). 


\section{$\underline{\text { 4. Simulation results }}$}

The hit rates for wind speed, wind direction and Temperature were calculated for every hour of the simulation using allowed deviations as given in Table 2. All hit rates are based on comparisons with the reference case and include all grid points of the model domain in Figure 1b; this are about $3 * 10^{6}$ grid points.

Table 2: Desired accuracy DA used for calculating the hit rate (from Cox et al., 1998).

\begin{tabular}{|l|c|c|c|c|c|}
\hline Variable & $\begin{array}{c}\text { Temperatur } \\
\mathbf{e}\left({ }^{\circ} \mathbf{C}\right)\end{array}$ & $\begin{array}{c}\text { Dew point } \\
\text { depression }\left({ }^{\circ} \mathbf{C}\right)\end{array}$ & $\begin{array}{c}\text { Wind speed } \\
\left(\mathbf{m ~ s}^{-1}\right)\end{array}$ & Wind direction & $\begin{array}{c}\text { Pressure } \\
(\mathbf{h P a})\end{array}$ \\
\hline Desired & \pm 2 & \pm 2 & $\begin{array}{c} \pm 1 \text { for ff }<10 \mathrm{~m} \mathrm{~s}^{-1} \\
\pm 2.5 \mathrm{for} \mathrm{ff}>10 \mathrm{~m} \mathrm{~s}^{-1}\end{array}$ & $\pm 30^{\circ}$ & \pm 1.7 \\
\hline accuracy DA & & & & \\
\hline
\end{tabular}

Values for the hit rates (in \%) are shown in Figure 2 for the six different simulations. The simulation "eu_6km_nonesting" has by far the largest deviations from the reference case. This shows that the situation is indeed sensitive to changes in the large-scale situation. In addition, also "eu_6km_ana" yields quite low hit rates. There are two probably reasons for this: a) the update interval of the forcing data is too small compared to the changes in the large-scale situation; b) the forcing data derived from the analysis are differ from the coarse grid simulation results and therefore lead to the low hit rates which are based on the results of the reference simulation.

The simulations nested in the coarse grid METRAS results agree in a similar way with the reference simulation in the second half of the simulation time (after about 21 hours; i.e. 18 CET of 29.08.2003. At this time the performance of simulation eu_6km_ana is somewhat closer to the other nested simulations than before. This might be a hint that the nesting becomes less relevant and the situation is more locally driven. In the first 21 hours of the simulation the two simulations with constant update interval (3 hours, 6 hours) are closest to the reference case, while the adaptive update simulations (eu_6km_lcout80, eu_6km_lcout20) show a high variability in performance. This is a hint that the acceleration is probably not a reliable measure to determine update intervals. The best performance is received in the present case study for a nesting every 3 hours. 
(a)

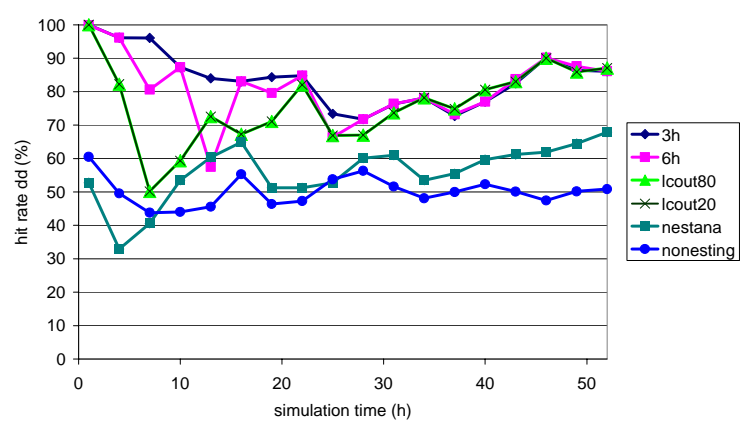

(c)

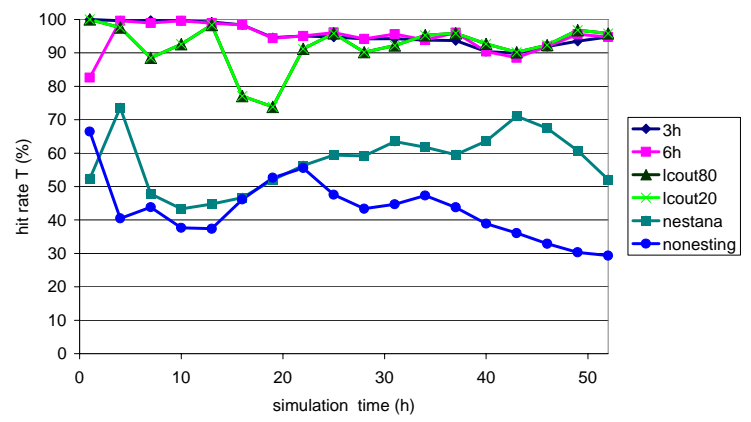

(b)

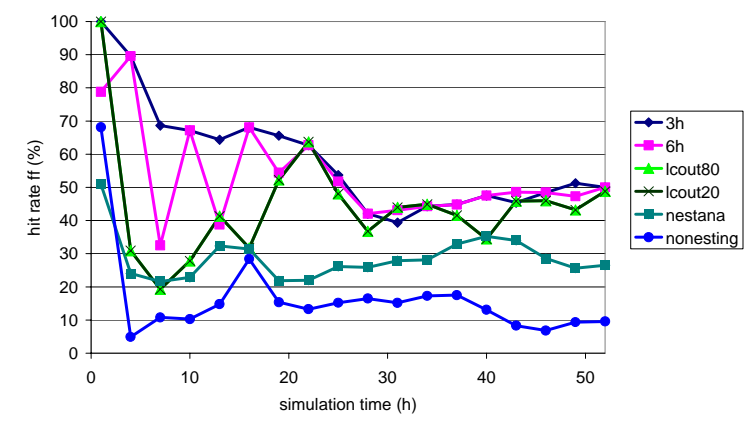

Figure 2: Hit rates for wind speed (a), wind direction (b) and temperature (c). The hit rates are calculated by comparing results of the simulations with the highresolution reference case.

\section{Conclusions and outlook}

METRAS is able to yield simulation results on different scales and in different detail. Nesting is a helpful tool to improve the model performance if the forcing data represent the meteorological situation in a realistic way. The current investigations on the impact of update interval lengths on model performance lead to the following conclusions:

a) In general, shorter update intervals lead to higher model performance.

b) Consecutive, but very different intervals seem to reduce the positive effect.

c) With increasing forecast time the uncertainty in the forecast that is resulting from the update of the boundary values seems to decrease.

Especially the last result is surprising, since it is generally assumed that for longer forecast with limited area models the boundary values become more and more relevant. It therefore needs to be investigated, if this is a result only true for the situation investigated or if it is more general.

A next step to further investigate the influence of different update intervals will be to define a characteristic time for the occurring changes and using this characteristic time to control the writing of the data that are used as forcing fields in the high resolution 
simulations. First results seem to be promising in enhancing model performance compared to the constant update interval of 3 hours.

\section{Acknowledgments:}

This work has been supported by the German Research Foundation (DFG) in the framework of the project SCHL499-2 and the Sonderforschungsbereich 512 „Cyclones and the North Atlantic Climate System“. This work is a contribution to COST728.

\section{References:}

Cox, R., B. L. Bauer and T. Smith (1998): Mesoscale model intercomparison. Bull. Am. Meteorol. Soc., 79, 265-283.

Lenz C.-J., Müller F. and Schlünzen K.H. (2000): The sensitivity of mesoscale chemistry transport model results to boundary values. Env. Monitoring and Assessment, 65, 287 -298.

López S.D., Lüpkes C. and Schlünzen K.H. (2005): The effects of different k-eclosures on the results of a micro-scale model for the flow in the obstacle layer. Meteorol. Zeitschrift, 14, 839-848.

Müller F., Schlünzen K.H. and Schatzmann M. (2000): Test of numerical solvers for chemical reaction mechanisms in 3D air quality models. Environmental Modelling Software, 15, 639-646.

Schlünzen, K. H. and J. J. Katzfey (2003): Relevance of subgrid-scale land-use effects for mesoscale models. Tellus 55A, 232-246.

Schlünzen K.H. (1990): Numerical studies on the inland penetration of sea breeze fronts at a coastline with tidally flooded mudflats, Beitr. Phys. Atmosph., 63, 243-256.

Trukenmüller A., Grawe D. and Schlünzen K.H. (2004): A model system ort he assessment of ambient air quality conforming to EC directives, Meteorol. Zeitschrift, Vol. 13, No. 5, 387-394.

Schlünzen K.H., Meyer E.M.I. (2007): Impacts of meteorological situations and chemical reactions on daily dry deposition of nitrogen into the Southern North Sea. Atmospheric Environment, 41-2, 289-302.

Schlünzen K.H., Hinneburg D., Knoth O., Lambrecht M., Leitl B., Lopez S., Lüpkes C., Panskus H., Renner E., Schatzmann M., Schoenemeyer T., Trepte S. and Wolke R. (2003): Flow and transport in the obstacle layer - First results of the microscale model MITRAS. J. Atmos. Chem., 44, 113-130. 